\title{
Global Attractor of a Predator-Prey System with Impulsive Effect and Control
}

\author{
Chen Ning ${ }^{1, *}$, Tian Baodan ${ }^{1}$, Chen Yang ${ }^{2}$ and Chen Jiqian ${ }^{3}$ \\ ${ }^{1}$ School of Science, Southwest University of Science and Technology, Mianyang, 621010, China \\ ${ }^{2}$ Audit office, Southwest University of Science and Technology, Mianyang, 621010, China \\ ${ }^{3}$ Southwest University of Science and Technology, Mianyang, 621010, China
}

\begin{abstract}
In this paper, the author discusses the Global attractor of solution for the boundary value problem of the dynamic system with impulsive case. Based on the prey-Predator with impulsive Effect and HollingIII functional response is proposed and analyzed. By using the Floquet theory of impulsive equation and comparison theorem, sufficient conditions for the system to extinct and some permanence arc given. Finally, the numerical simulation was introduced to support these excellent extensions of results. The authors extend dynamic behavior and the critical value to continuum more previous work [3, 7, 8, 9 and 10].
\end{abstract}

Keywords: Biological control, attractive, predator-Prey system, impulsive effect.

\section{INTRODUCTION}

The problem of Attractor in dynamic system is not only to examine foundation of solution in the corresponding system, and it is also to study well-pseudo-plastic with stability, branch, period, extinction and blow-up of solutions, and chaotic phenomenon premise, it is to examine the solution as an important method (See [1-10]). In applications, we utilize these problems to study and discussion that species permanence and development, environmental protection, atmospheric turbulence and the strong convective phenomenon case are an important investigation role [1-3].

\section{MODEL INTRODUCED}

We have a sigmoid functional response in fooddependent digestion model, consider a more general situation

where, the parameters $a, b, c, d, e, f, r, h_{i}, \lambda_{i}, d_{i}, b_{i}, i=1,2,3,4$ are the normal number, $i=1,2,3,4 k=1,2,3 ; 1 \leq \alpha \leq 3$ when $k=2, \alpha \equiv 1, \lambda_{2}=\lambda_{3}=\lambda_{4}=0, \quad d_{2}=d_{3}=d_{4}=0 \quad$, $b_{2}=b_{3}=b_{4}=0, z(t)=\omega(t)=\eta(t)=0$, Sigmoid functional response to food-dependent digestion model [3].

$$
\left\{\begin{array}{l}
x^{\prime}(t)=x(t)\left(r-a x^{\alpha}(t)-b y(t)-c z(t)-d \omega(t)-e \eta(t)\right) \\
y^{\prime}(t)=y(t)\left(\lambda_{1} b_{1} x^{k}(t) /\left(1+h_{1} b_{1} x^{k}(t)\right)-d_{1}\right) \\
z^{\prime}(t)=z(t)\left(\lambda_{2} b_{2} x^{k}(t) /\left(1+h_{2} b_{2} x^{k}(t)\right)-d_{2}\right) \\
\omega^{\prime}(t)=\omega(t)\left(\lambda_{3} b_{3} x^{k}(t) /\left(1+h_{3} b_{3} x^{k}(t)\right)-d_{3}\right) \\
\eta^{\prime}(t)=\eta(t)\left(\lambda_{4} b_{4} x^{k}(t) /\left(1+h_{4} b_{4} x^{k}(t)\right)-d_{4}\right)
\end{array}\right.
$$

The models of (2.1) are assumption as follow:

1. No Predator, the prey is logistic growth.

2. Four kinds of predator and a prey with relation in direct proportion to that $-b_{1} x(t) y(t),-b_{2} x(t) z(t),-b_{3} x(t) \omega(t)$,

$-b_{4} x(t) \eta(t)$

3. All four types of prey in the absence, resulting in the form of mortality index, which is $-d_{1} y(t),-d_{2} z(t),-d_{3} \omega(t),-d_{4} \eta(t)$.

4. Four types of predator on prey growth rate is $\lambda_{1} b_{1} x^{k}(t) y(t) /\left(1+h_{1} b_{1} x^{k}(t)\right), \lambda_{2} b_{2} x^{k}(t) z(t) /\left(1+h_{2} b_{2} x^{k}(t)\right)$,

$$
\begin{gathered}
\lambda_{3} b_{3} x^{k}(t) \omega(t) /\left(1+h_{3} b_{3} x^{k}(t)\right) \\
\lambda_{4} b_{4} x^{k}(t) \eta(t) /\left(1+h_{4} b_{4} x^{k}(t)\right),
\end{gathered}
$$

which $b_{1}, b_{2}, b_{3}, b_{4}$ for predator search rate of prey, predator $h_{1}, h_{2}, h_{3}, h_{4}$ are four kinds of prey, respectively, digestion time, $\lambda_{1}, \lambda_{2}, \lambda_{3}, \lambda_{4}$ is the predator, respectively, the four digestion rate of prey species.

In fact, after a simple calculation shows that the model (2.1) to the classical Lotka-Volterra model has the same line, such as dumping.

We assume that:

$$
\begin{aligned}
\min & \left\{\left(\lambda_{1} b_{1}-d_{1} b_{1} h_{1}\right)^{\frac{\alpha}{k}},\left(\lambda_{2} b_{2}-d_{2} b_{2} h_{2}\right)^{\frac{\alpha}{k}},\left(\lambda_{3} b_{3}-d_{3} b_{3} h_{3}\right)^{\frac{\alpha}{k}},\left(\lambda_{4} b_{4}-d_{4} b_{4} h_{4}\right)^{\frac{\alpha}{k}}\right\} \\
& >\frac{a}{r} \max \left\{d_{1}, d_{2}, d_{3}, d_{4}\right\}
\end{aligned}
$$

then the system (2.1) there is a balance point 
$\left(0, y^{\prime}(t), z^{\prime}(t), \dot{\omega}^{*}(t), \eta^{\prime}(t)\right)$.

The introduction of periodic pulse injected to kill pests and natural enemies to control pest populations to achieve balance. To this end digestion of food-dependent model (2.1) to improve the time for the pulse to give with impulsive differential equation

$$
\left\{\begin{array}{l}
x^{\prime}(t)=x(t)\left(r-a x^{\alpha}(t)-b y(t)-c z(t)-d \omega(t)-e \eta(t)\right) \\
y^{\prime}(t)=y(t)\left(\lambda_{1} b_{1} x^{k}(t) /\left(1+h_{1} b_{1} x^{k}(t)\right)-d_{1}\right) \\
z^{\prime}(t)=z(t)\left(\lambda_{2} b_{2} x^{k}(t) /\left(1+h_{2} b_{2} x^{k}(t)\right)-d_{2}\right) \\
\omega^{\prime}(t)=\omega(t)\left(\lambda_{3} b_{3} x^{k}(t) / 1+h_{3} b_{3} x^{k}(t)-d_{3}\right) \\
\eta^{\prime}(t)=\eta(t)\left(\lambda_{4} b_{4} x^{k}(t) /\left(1+h_{4} b_{4} x^{k}(t)\right)-d_{4}\right) \\
\Delta x(t)=-p_{1} e^{\theta x(t)} \\
\Delta y(t)=-p_{2} y(t)+\tau_{1} \\
\Delta z(t)=-p_{3} z(t)+\tau_{2} \\
\Delta \omega(t)=-p_{4} \omega(t)+\tau_{3} \\
\Delta \eta(t)=-p_{5} \eta(t)+\tau_{4}
\end{array}\right\} t \neq n T
$$

where

$\Delta x(t)=x\left(t^{+}\right)-x(t), \Delta y(t)=y\left(t^{+}\right)-y(t), \Delta z(t)=z\left(t^{+}\right)-z(t)$,

$\Delta \omega(t)=\omega\left(t^{+}\right)-\omega(t)$ and $\Delta \eta(t)=\eta\left(t^{+}\right)-\eta(t)$

$\left(\tau_{i} \geq 0, i=1,2,3,4\right)\left(0 \leq p_{j}<1, j=1,2,3,4,5\right), e^{\theta x(t)}=\theta(1+R \theta) x(t)$, here $R \theta^{2} x(t)=\left(2+(\xi x(t))^{2}\right) / 2, n \in N, N \geq 0$ integer set, the T implies periodic impulsive.

\section{DEFINITIONS AND LEMMAS}

Set $R_{+}=[0, \infty), R_{+}^{5}=\left\{\sigma \in R^{5} \mid \sigma \geq 0\right\}$. Function $f=\left(f_{1}, f_{2}, f_{3}, f_{4}, f_{5}\right)^{T}$, which are the equations $f_{1}, f_{2}, f_{3}, f_{4}, f_{5}$ of (2.2) give the function equation of the right end of (2.2). Assume that

$$
\begin{aligned}
V_{0}=\left\{V \mid R_{+} \times R_{+}^{5} \rightarrow R_{+}, V \in C(n T,(n+1) T],\right. \\
\left.V(t, u) \stackrel{(t, u) \rightarrow\left(n T^{+}, \sigma\right), t>n T}{\longrightarrow} V\left(n T^{+}, \sigma\right)\right\}
\end{aligned}
$$

Definition 2.1 Set $V \in V_{0} \quad, \quad$ Then $(t, \sigma) \in(n T,(n+1) T)] \times R_{+}^{5}$, on systems $(2.2)$ is defined as the upper right derivative

$D^{+} V(t, \sigma)=\lim _{h \rightarrow 0^{+}} \sup [V(t+h, \sigma+h f(t, \sigma))-V(t, \sigma)] / h^{\bullet}$
Lemma 2.2 Set $\sigma(t)$ is solution of the initial value of the system (2.2) with $\sigma\left(0^{+}\right) \geq 0$, then if for arbitrary $t \geq 0$, we have $\sigma(t) \geq 0$. Thus we obtain $\sigma(t)>0, t \geq 0$, for the $\sigma\left(0^{+}\right)>0$, here $\sigma(t)=(x(t), y(t), z(t), \omega(t), \eta(t))$.

Proof: easy to have

$$
\begin{aligned}
x(t) & =x\left(0^{+}\right)(1-\theta(1+R \theta) p)^{m} \\
& \times \exp \left(\int_{0}^{t}\left(r-a x^{\alpha}(s)-b y(s)-c z(s)-d \omega(s)-e \eta(s)\right) d s\right), \\
y(t) & =y\left(0^{+}\right) \exp \left(\int_{0}^{t}\left(\lambda_{1} b_{1} x^{k}(s) /\left(1+h_{1} b_{1} x^{k}(s)\right)-d_{1}\right) d s\right) \\
& +\tau_{1} \sum_{i=1}^{4} \int_{i T}^{t}\left(\lambda_{i} b_{i} x^{k}(s) /\left(1+h_{i} b_{i} x^{k}(s)\right)-d_{i}\right) d s,
\end{aligned}
$$

which the $m$ is in the interval $[0, t]$ of the pulse frequency, $t \in[0,+\infty)$ is arbitrary, in the same way, for $z(t), \omega(t)$,

$\eta(t)$, by above same calculating method that we obtain similar result.

Definition 2.3 If $(x(t), y(t), z(t), \omega(t), \eta(t))$ is solution of system (2.2) satisfy $x\left(0^{+}\right), y\left(0^{+}\right), z\left(0^{+}\right), \omega\left(0^{+}\right)$and $\eta\left(0^{+}\right)>0$. when there is constants $M \geq m>0$ such that $m \leq x(t) \leq M, m \leq y(t) \leq M, \mathrm{~L}, m \leq \eta(t) \leq M,(2.3)$ then the system (2.2) is called uniformly persistence.

We consider the following system of the nature of extinction,

$\left\{\begin{array}{l}d \sigma / d t=-p \sigma(t), t \neq n T, \\ \Delta \sigma(t)=-p \sigma(t)+\tau, t=n T .\end{array}\right.$

Lemma 2.3 System (2.4) there is a global asymptotic stability of positive periodic solution:

$\sigma^{*}(t)=\tau e^{-p(t-n T)} /\left(1-(1-P) e^{-p T}\right), t \in(n T,(n+1) T], n \in N$, where, the initial value of $\sigma^{*}\left(0^{+}\right)=\tau /\left(1-(1-P) e^{-p T}\right)$

From lemma 2.3, we may get bellow lemma.

Lemma 2.4 System (2.2) there is a predator eradication periodic solution, that is

$\left(0, y^{*}(t), z^{*}(t), \omega^{*}(t), \eta^{*}(t)\right)$

$$
\begin{aligned}
& =\left(\begin{array}{l}
0, \frac{\tau_{1} e^{-p_{2}(t-n T)}}{1-\left(1-p_{2}\right) e^{-p_{1} T}}, \frac{\tau_{2} e^{-p_{3}(t-n T)}}{1-\left(1-p_{3}\right) e^{-p_{2} T}}, \\
\frac{\tau_{3} e^{-p_{3}(t-n T)}}{1-\left(1-p_{4}\right) e^{-p_{1} T}}, \frac{\tau_{4} e^{-p_{4}(t-n T)}}{1-\left(1-p_{5}\right) e^{-p_{1} T}}
\end{array}\right), \\
& t \in(n T,(n+1) T], n \in N .
\end{aligned}
$$


We use the literature [2], Theorem 3.1.1] the methods and results are given the following lemma

Lemma 2.5 [3] (comparison theorem) Let $V \in V_{0}$ to satisfy the following inequality

$\begin{cases}D^{+} V(t, \sigma) \leq h(t, V(t, \sigma)), & t \neq n T, \\ V\left(t, \sigma\left(t^{+}\right)\right) \leq \psi_{n}(t, V(t, \sigma)), & t=n T,\end{cases}$

Which $h: R_{+}^{5} \rightarrow R$ satisfy in the Theorem 3.1.1 [2]. Assumption that $h: R_{+} \times R_{+}^{5} \rightarrow R_{+}$in $(n T,(n+1) T] \times R_{+}^{5}$ continuous, and the $\sigma \in R_{+}^{5}, n \in N$, there exists $h(t, u) \rightarrow h\left(n T^{+}, \sigma\right)$, as

$(t, u) \rightarrow\left(n T^{+}, \sigma\right)$. Here $\psi_{n}: R_{+} \rightarrow R_{+}$is non-decrease function. Let $r(t)$ is the maximal

solution on $[0,+\infty)$ in the following scalar impulsive differential equation.

$$
\left\{\begin{array}{l}
u^{\prime}=h(t, u(t)), \quad t \neq n T, \\
u\left(t^{+}\right)=\psi_{n}(u(t)), t=n T \\
u\left(0^{+}\right)=u_{0}
\end{array}\right.
$$

Then $V\left(0^{+}, \sigma_{0}\right) \leq u_{0}$. So $V(t, \sigma(t)) \leq r(t), t \geq 0, \sigma(t)$ is the solution of (2.2).

We give the fundamental nature of subsystems of the (2.2)

$$
\begin{aligned}
& \left\{\begin{array} { c } 
{ y ^ { \prime } = - d _ { 1 } y ( t ) , t \neq n T , } \\
{ y ( t ^ { + } ) = ( 1 - p _ { 2 } ) y ( t ) + \tau _ { 1 } , } \\
{ \quad t = n T , } \\
{ y ( 0 ^ { + } ) = y _ { 0 } . }
\end{array} \left\{\begin{array}{c}
z^{\prime}=-d_{2} z(t), t \neq n T, \\
z\left(t^{+}\right)=\left(1-p_{3}\right) z(t)+\tau_{2},, \mathrm{~L}, \\
t=n T, \\
z\left(0^{+}\right)=z_{0} .
\end{array},\right.\right. \\
& \left\{\begin{array}{l}
\eta^{\prime}=-d_{4} z(t), t \neq n T, \\
\eta\left(t^{+}\right)=\left(1-p_{5}\right) \eta(t)+\tau_{4}, \\
\quad t=n T, \\
\eta\left(0^{+}\right)=\eta_{0} .
\end{array}\right.
\end{aligned}
$$

(2.7) of (2.2) has a positive periodic solution $y^{*}(t), z^{*}(t)$, $\omega^{*}(t), \eta^{*}(t)$, and the any other solution

$y(t), z(t), \omega(t), \eta(t)$ of the subsystems (2.7), we have $\left|y(t)-y^{*}(t)\right| \rightarrow 0,\left|z(t)-z^{*}(t)\right| \rightarrow 0,\left|\omega(t)-\omega^{*}(t)\right| \rightarrow 0,\left|\eta(t)-\eta^{*}(t)\right| \rightarrow 0$ as when $t \rightarrow \infty$. where

$$
\begin{aligned}
& y^{*}(t)=\tau_{1} e^{-d_{1}(t-n T)} /\left(1-\left(1-p_{2}\right) e^{-d_{1} T}\right), t \in(n T,(n+1) T], n \in N, \\
& y^{*}\left(0^{-}\right)=\tau_{1} /\left(1-\left(1-p_{2}\right) e^{-d_{1} T}\right), \\
& z^{*}(t)=\tau_{2} e^{-d_{2}(t-n T)} /\left(1-\left(1-p_{3}\right) e^{-d_{2} T}\right), t \in(n T,(n+1) T], n \in N, \\
& z^{*}\left(0^{-}\right)=\tau_{2} /\left(1-\left(1-p_{3}\right) e^{-d_{2} T}\right), \cdots, \\
& \eta^{*}(t)=\tau_{4} e^{-d_{4}(t-n T)} /\left(1-\left(1-p_{5}\right) e^{-d_{4} T}\right), t \in(n T,(n+1) T], n \in N, \\
& \eta^{*}\left(0^{-}\right)=\tau_{4} /\left(1-\left(1-p_{5}\right) e^{-d_{4} T}\right) .
\end{aligned}
$$

\section{MAIN RESULTS}

We will give control of the conditions of Prey $x(t)$ extinction.

Theorem 3.1 The system set $(x(t), y(t), z(t), \omega(t), \eta(t))$ (2.2) of any one solution, if

$$
T<r^{-1} \ln \left(1 /\left(1-\theta p_{1}\right)\right)+r^{-1} \sum_{i=1}^{4}\left(b_{i} \tau_{i} / r d_{i}\left(1-P_{i+1}\right)\right) \stackrel{\Delta}{=} T_{\max }
$$

established, Periodic Solutions of the predator extinction $\left(0, y^{*}(t), z^{*}(t), \omega^{*}(t), \eta^{*}(t)\right)$ is globally asymptotically stable.

Proof : First, we consider the small perturbation solution of periodic solutions identify the local stability. Our defini-

tion of $\quad x(t)=u_{1}(t), y(t)=y^{*}(t)+u_{2}(t), \cdots$, $\eta(t)=\eta^{*}(t)+u_{s}(t)$,

then we have

$$
\left(\begin{array}{l}
u_{1}(t) \\
\mathrm{M} \\
u_{5}(t)
\end{array}\right)=\Phi(t)\left(\begin{array}{l}
u_{1}(0) \\
\mathrm{M} \\
u_{5}(0)
\end{array}\right), 0 \leq t<T .
$$

where $\Phi$ to satisfy the following equation

$$
\frac{d \Phi(t)}{d t}=\left(\begin{array}{cccc}
r-b y^{*}(t)-c z^{*}(t)-d \omega^{*}(t)-e \eta^{*}(t) & 0 & \mathrm{~L} & 0 \\
b_{1} y^{*}(t) & -d_{1} & 0 & \mathrm{M} \\
\mathrm{M} & 0 & 0 & 0 \\
b_{4} \eta^{*}(t) & 0 & 0 & -d_{4}
\end{array}\right) \Phi(t)
$$

$\Phi(0)=I$ is a unit matrix. From the above equation can be easily find

$$
\Phi(t)=\left(\begin{array}{cccc}
\exp \left(\int_{0}^{t}\left(r-b y^{*}(s)-c z^{*}(s)-d y^{*}(s)-e z^{*}(s)\right) d s\right) & 0 & \mathrm{~L} & 0 \\
\Delta & e^{-d_{1} t} & \mathrm{~L} & 0 \\
\mathrm{M} & \Delta & 0 & 0 \\
\Delta & \Delta & \Delta & e^{-d_{d^{t}}}
\end{array}\right)
$$

This brings me to sign " $\Delta$ " items to determine the form of the following does not affect our analysis, not calculated. 
By for $t=n T$ case of system (2 .2) reduce into the linear equation as

$$
\left(\begin{array}{l}
u_{1}\left(n T^{+}\right) \\
u_{2}\left(n T^{+}\right) \\
\mathrm{M} \\
u_{5}\left(n T^{+}\right)
\end{array}\right)=\left(\begin{array}{cccc}
1-\theta(1+R \theta) p_{1} & 0 & \mathrm{~L} & 0 \\
0 & 1-p_{2} & 0 & \mathrm{M} \\
\mathrm{M} & 0 & 0 & 0 \\
0 & \mathrm{~L} & 0 & 1-p_{5}
\end{array}\right)\left(\begin{array}{l}
u_{1}(n T) \\
u_{2}(n T) \\
\mathrm{M} \\
u_{5}(n T)
\end{array}\right) .
$$

We are T periodic pulse from the linear differential equations by Floquet theory [1] that $\left(0, y^{*}(t), z^{*}(t), \omega^{*}(t), \eta^{*}(t)\right)$ is the stability of periodic solutions given by the following single-valued matrix $M$ defined by eigenvalues.

Therefore, if the matrix

$M=\left(\begin{array}{cccc}1-\theta(1+R \theta) p_{1} & 0 & \cdots & 0 \\ 0 & 1-p_{2} & \ddots & \vdots \\ \vdots & \ddots & \ddots & 0 \\ 0 & \cdots & 0 & 1-p_{5}\end{array}\right) \Phi(T)=$

$$
\left(\begin{array}{cccc}
m_{11} & 0 & \cdots & 0 \\
* & m_{22} & \ddots & \vdots \\
* & * & \ddots & 0 \\
* & * & * & m_{55}
\end{array}\right)
$$

Where in above matrix that the diagonal elements; $\mu_{i}=m_{i i},(i=1,2, \mathrm{~L} 5)$. If the five eigenvalues of modulus less than 1 , then the cycle is a partial solution $\left(0, y^{*}(t), z^{*}(t), \omega^{*}(t), \eta^{*}(t)\right)$ stable. In fact, the five Floquet multipliers are

$\mu_{1}=\left(1-\theta(1+R \theta) p_{1}\right)\left(\exp \int_{0}^{t}\left(r-b y^{*}(s)-c z^{*}(s)-d \omega^{*}(s)-e \eta^{*}(s)\right) d s\right)$,

and $\mu_{j}=\left(1-p_{j}\right) e^{-d_{j-1} t}, j=2,3,4,5$, according to Floquet theory, if $\left|\mu_{i}\right|<1, i=1,2,3,4,5$, that is,

$$
T<r^{-1} \ln \left(1 /\left(1-\theta p_{1}\right)\right)+r^{-1} \sum_{i=1}^{4}\left(b_{i} \tau_{i} / d_{i}\left(1-P_{i+1}\right)\right)
$$

System (2.2) Periodic Solutions $\left(0, y^{*}(t), z^{*}(t), \omega^{*}(t), \eta^{*}(t)\right)$ is a local stable.

Next, proof the $\left(0, y^{*}(t), z^{*}(t), \omega^{*}(t), \eta^{*}(t)\right)$ is global attractor. tion

We consider the following impulsive differential equa-

$\left\{\begin{array}{l}v^{\prime}=-d v(t), t \neq n T, \Delta v(t)=v\left(t^{+}\right)-v(t)=-p v(t)+\tau, t=n T, \\ v\left(0^{+}\right)=\sigma\left(0^{+}\right)\end{array}\right.$

By Lemma 2.2 and Lemma 2.3, to be $y(t), z(t), \omega(t), \eta(t) \geq u(t)$, and $v(t) \rightarrow y^{*}(t)$, as $t \rightarrow \infty$.
So $y^{*}(t)-\varepsilon<v(t) \leq y(t)$, then for $z(t), \omega(t), \eta(t)$ by above same method for all $t \geq 0$ that similar conclusion.

From the system (2 .2) available

$\left\{x^{\prime}(t) \leq x(t)\left(r-b\left(y^{*}(t)-\varepsilon\right)-c\left(z^{*}(t)-\varepsilon\right)-d\left(\omega^{*}(t)-\varepsilon\right)-e\left(\eta^{*}(t)-\varepsilon\right)\right), t \neq n T,\left(^{*}\right)\right.$ $x\left(t^{+}\right)=\left(1-\theta p_{1}\right) x(t), t=n T$.

Use Lemma 2.3, easy access to

$x((n+1) T)$

$\leq x\left(n T^{+}\right) \exp \left(\int_{n T}^{(n+1) T}\left(r-b\left(y^{*}(t)-\varepsilon\right)-c\left(z^{*}(t)-\varepsilon\right)-d\left(\omega^{*}(t)-\varepsilon\right)-e\left(\eta^{*}(t)-\varepsilon\right)\right) d t\right)$

$=x(n T)\left(1-\theta p_{1}\right)$

$\times \exp \left(r T-b\left(r / d_{1}\right)-c\left(r / d_{2}\right)-d\left(r / d_{3}\right)-e\left(r / d_{4}\right)-4 \varepsilon T\right)$

As a result of $x(n T) \leq x\left(0^{+}\right) \delta^{n}$ and $x(n T) \stackrel{n \rightarrow \infty}{\rightarrow} 0$. Because $0<x(t) \leq x(n T)\left(1-\theta p_{1}\right) e^{r T}$

for $n T<t \leq(n+1) T$. So $x(t) \rightarrow 0$ when $n \rightarrow \infty$.

Theorem 3.2 Assume that constant $M>0$, such that for any a solution of system (2.1) with $\sigma(t)=(x(t), y(t), z(t), \omega(t), \eta(t))$, as time $t$ enough large, that

$\max \{x(t), y(t), z(t), \omega(t), \eta(t)\} \leq M$

Proof: Let $\sigma(t)=(x(t), y(t), z(t), \omega(t), \eta(t))$ for with any a solution of system (2.1).

Assume

$V(t)=\lambda_{1} x(t) / 2 \sqrt{h_{1} b_{1}}+y(t)+z(t)+\omega(t)+\eta(t)$, then $V(t) \in V_{0}=V\left(t_{0}\right)$ and imply

$D^{+} V(t)+C V(t)$

$=\frac{\lambda_{1} x(t)\left(r-a x^{\alpha}(t)-b y(t)-c z(t)-d \omega(t)-e \eta(t)\right.}{2 \sqrt{h_{1} b_{1}}}$

$+c\left(\left(\lambda_{1} x(t) / 2 \sqrt{h_{1} b_{1}}\right)+y(t)+z(t)+\omega(t)+\eta(t)\right)$

$\leq \frac{\lambda_{1}(r+c) x(t)}{2 \sqrt{h_{1} b_{1}}}-\frac{\lambda_{1} a x^{\alpha+1}(t)}{2 \sqrt{h_{1} b_{1}}}-\frac{\lambda_{1} b x(t) y(t)}{2 \sqrt{h_{1} b_{1}}}-\frac{\lambda_{1} c x(t) z(t)}{2 \sqrt{h_{1} b_{1}}}$

$-\frac{\lambda_{1} d x(t) \omega(t)}{2 \sqrt{h_{1} b_{1}}}-\frac{\lambda_{1} \operatorname{ex}(t) \eta(t)}{2 \sqrt{h_{1} b_{1}}}-\left(d_{1}-c\right) y(t)-\left(d_{2}-c\right) z(t)$

$-\left(d_{3}-c\right) \omega(t)-\left(d_{4}-c\right) e \eta(t), t \neq n T,(4.4)$

$V\left(n T^{+}\right)=\lambda_{1} x(n T) / 2 \sqrt{h_{1} b_{1}}+y(n T)+z(n T)+\omega(n T)+\eta(n T)+\tau$

Clearly, when $0<c<\min \left\{d_{1}, d_{2}, d_{3}, d_{4}\right\}$, the first inequality (4.4) is bounded, and choose these constants $c_{0}, M_{0}, \tau=\tau\left(\tau_{1}, \tau_{2}, \tau_{3}, \tau_{4}\right)$.So, the (4.4) reduces

$\left\{\begin{array}{l}D V(t) \leq-c_{0} V(t)+M_{0}, t \neq n T, \\ V\left(n T^{+}\right) \leq V(n T)+\tau, t=n T .\end{array}\right.$ 
By lemma (3.4) in [3], we get

$V(t) \leq\left(V\left(0^{+}\right)-M_{0} / c_{0}\right) e^{-c_{0} t}+\tau\left(\left(1-e^{-n c_{0} T}\right) /\left(1-e^{-c_{0} T}\right)\right) e^{-c_{0}(t-n T)}+M_{0} / c_{0}$,

where $t \in(n T,(n+1) T)$.Thus $V(t)$ is essential bounded, and there is constant $M>0$, such that any a solution of (2.1) with $\sigma(t)=(x(t), y(t), z(t), \omega(t), \eta(t))$, if $t$ enough large, it is also hold $\max \{x(t), y(t), z(t), \omega(t), \eta(t)\} \leq M$.

Remark: The further we can along with the way in [3], the system (2.2) can be the persistence by

$\left.r T-\sum_{i=1}^{4}\left(b_{i} \tau_{i} / d_{i}\left(1-p_{i+1}\right)\right)\right)>\ln \left(1-\theta p_{1}\right)^{-1}$.

\section{NUMERICAL COMPUTATION}

The Numerical simulations have been carried out to substantiate our analytical findings and investigate the global dynamical behavior of the nonlinear system (2.1).In the previous sections, the qualitative analyses of system are presented in Predator-Prey systems. Now, to see dynamical behavior of the system by Fig 1(a), (b), (c), and Fig. 2 (a), (b), (c).

For convenience, only consider three species with impulsive case for us as the following set of parameters: $\theta=0, \mathrm{r}=3$ $, \mathrm{k}=1, \mathrm{a}=0.2, \mathrm{~b}_{1}=0.75, \mathrm{~b} 2=0.9, \mathrm{c}_{1}=0.9, \mathrm{c}_{2}=0.85$, $\mathrm{d}_{1}=0.2, \mathrm{~d}_{2}=0.18, \mathrm{~h}_{1}=0.8, \mathrm{~h}_{2}=0.9$. Assume that initial value $\sigma_{0}=(2,0.1,0.1)$, by condition of above theorem 3.1 and theorem 3.2, and computing that critical value $T_{\max } \approx 4.000$ When impulsive periodic $T<T_{\max } \approx 4.000$ for action that the prey can be eradicated (see Fig.1 (a) (b) (c)). If it is with impulsive periodic $T>T_{\max } \approx 4.000$, that occur complex dynamic action of the periodic coexistence, and strange attractor. etc. (see Fig. (2a) (b) (c).

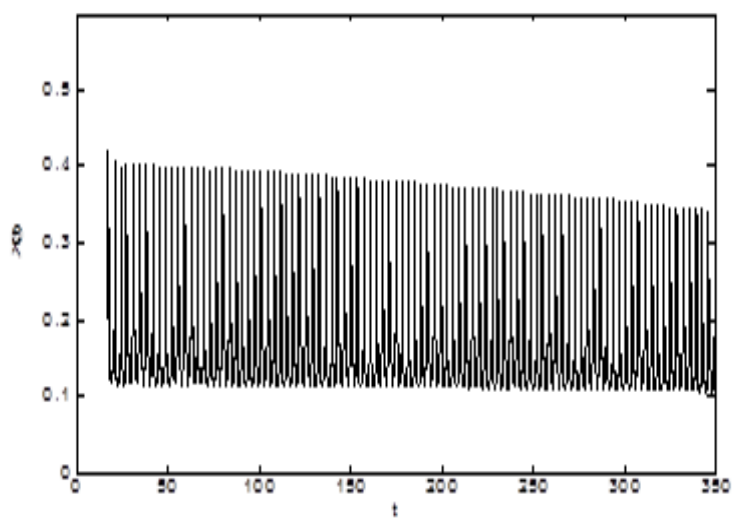

Fig. 1(a). Figure description case for pest species with parameters $\mathrm{r}=3, \mathrm{k}=1, \mathrm{a}=0.2, \mathrm{~b} 1=0.75, \mathrm{~b} 2=0.9, \quad \mathrm{c} 1=0.9, \quad \mathrm{c} 2=0.85, \mathrm{~d} 1=0.2$, $\mathrm{d} 2=0.18 ; \mathrm{h} 1=0.8 ; \mathrm{h} 2=0.9$. When the impulsive periodic $\mathrm{T}=3.5$, the prey species $\mathrm{x}(\mathrm{t})$ with decrease case for changing time.

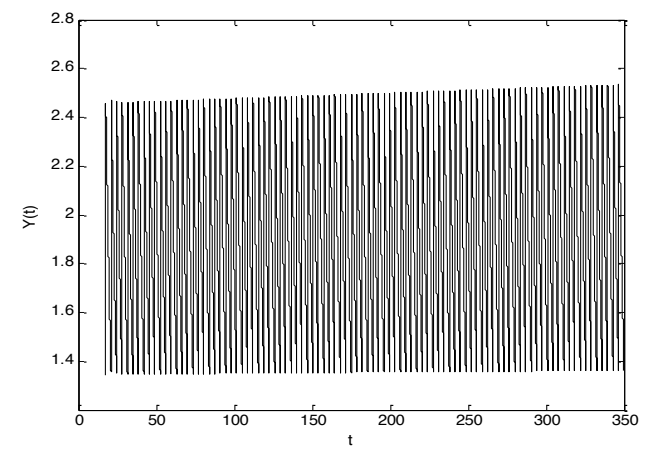

Fig.1(b). Figure description implies change of nature enemies species $y(t)$ with increase for changing time.

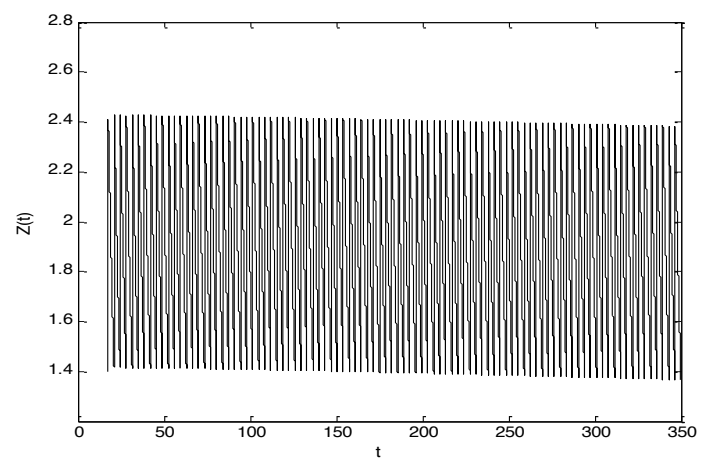

Fig.1(c). Figure description the change of nature enemies species $\mathrm{z}(\mathrm{t})$ with decrease for changing time.

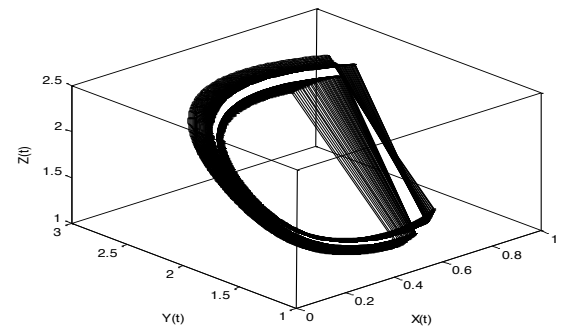

Fig. (2A). The system (2.2) has an chaos attractor, parameters with $\mathrm{r}=3, \mathrm{k}=1, \mathrm{a}=0.2, \quad \mathrm{~b} 1=0.75, \quad \mathrm{~b} 2=0.9, \quad \mathrm{c} 1=0.9, \quad \mathrm{c} 2=0.85, \quad \mathrm{~d} 1=0.2$, $\mathrm{d} 2=0.18, \mathrm{~h} 1=0.8, \mathrm{~h} 2=0.9$. When the impulsive periodic $\mathrm{T}=5.5$, the prey species $\mathrm{x}(\mathrm{t})$ with decrease case for changing time.

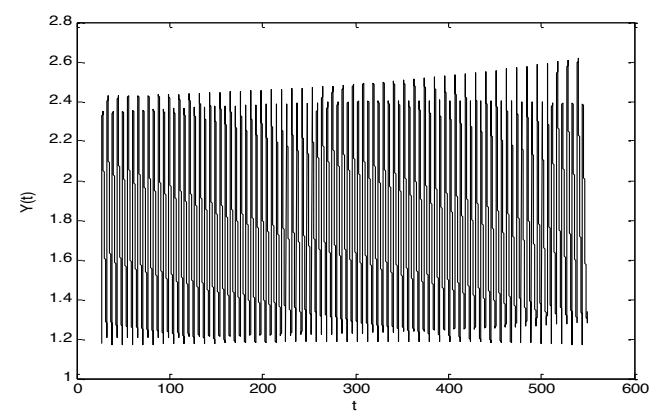

Fig. (2b). Implies change of nature enemies species y(t) along time. 


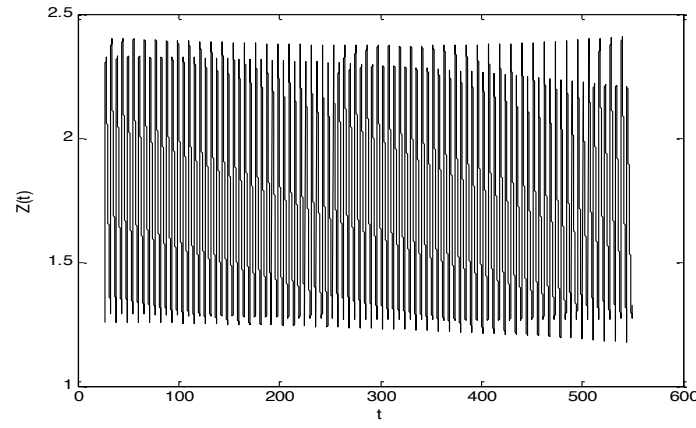

Fig. 2(c). Shows the branch of pest and nature enemies $z(t)$.

\section{CONCLUSION}

This article mainly studies the general dynamics behaviour of Burger-equation of blasting and extinguishing phenomenon, to discuss these cases through certain parameters values. Such equations always exhibit a rich phenomenology attracts many attention in engineering mechanics, material mechanics and fluid mechanics with application value. The authors extend dynamic behaviour and the critical value to continuum more previous work [3, 8, 10] for in-depth achieve for apply value.

\section{CONFLICT OF INTEREST}

The authors confirm that this article content has no conflict of interest.

\section{ACKNOWLEDGEMENTS}

This work is supported by the Natural Science Foundation (No.11ZB192) of Sichuan Education Bureau and the key program of Science and Technology Foundation
(No.11Zd1007) of Southwest University of Science and Technology.

\section{REFERENCES}

[1] Bainov D D, Simeonov P S Impulsive, Differential Equations: Periodic Solutions and Applications. New York, John Wiley and Sons, pp:143-167, 1993.

[2] Funasaki E, Ket M, "Invasion and chaos in a periodically pulsed mass-action chemostat," Theoretical population Biology, 44, pp:203-224, 1993.

[3] Zhand. S. W. and Chen. L. S, "Predator-Prey System With Impulsive Effect and Integrated Pest Control," J. Sys. and Math .Scis. 25, pp: 264-275, 2005.

[4] Lakshimilkantham V, Bainov D.D. Simeonov P.S "Theory of impulsive differential equations," Singapore world Scientific, pp: 350-374, 1989.

[5] Guo. D. J, "Positive Solutions of an infinite boundary value problem for nth-order nonlinear impulsive singular integraldifferential equations in Banach spaces," Nonlinear analysis, 70, pp:2078-2090, 2009.

[6] Chen H, Sun J, "An application of variational method to secondorder impulsive differential equation on the half line, "Appl Math Comput, 217, pp:1863-1869, 2010.

[7] Chen.Y. P, "The Dynamics of a Three-species Predator-prey System with Impulsive Effect," Mathematics in practice and theory, 39, pp:135-141, 2009.

[8] Chen. N, Jiqiang Chen. J.Q., "Operator equation and application of variation iterative method," Applied Mathematics, 3, pp: $857-$ 863, 2012.

[9] Chen. N, Tian. B.D., Chen. J.Q, "Blow-up of solutions for some a class of generalized nonlinear Schrodinger equations, " Appl. Math. Inf. Sci. 6-3S, pp:881-886, 2012.

[10] Ning Chen. N, Chen. J. Q, Tian. B. D, "Fixed Point Theorems of Multi-value map And He's Variation Iteration," Appl. Math. Inf. Sci. 6-3S, pp: 907-914, 2012.

Received: September 16, 2014

Revised: December 23, 2014

Accepted: December 31, 2014

(C) Ning et al.; Licensee Bentham Open.

This is an open access article licensed under the terms of the Creative Commons Attribution Non-Commercial License (http://creativecommons.org/licenses/by-nc/3.0/) which permits unrestricted, non-commercial use, distribution and reproduction in any medium, provided the work is properly cited. 